\title{
Análise crítica da interculturalidade na Política Nacional de Atenção às Populações Indígenas no Brasil
}

\author{
Leo Pedrana, ${ }^{1}$ Leny Alves Bomfim Trad, ${ }^{1}$ Maria Luiza Garnelo Pereira, ${ }^{2}$ \\ Mônica de Oliveira Nunes de Torrenté e Sara Emanuela de Carvalho Mota ${ }^{1}$
}

Como citar Pedrana L, Trad LAB, Pereira MLG, Torrenté MON, Mota SEC. Análise crítica da interculturalidade na Política Nacional de Atenção às Populações Indígenas no Brasil. Rev Panam Salud Publica. 2018;42:e178. https://doi.org/10.26633/RPSP.2018.178

RESUMO A preocupação com um cuidado culturalmente apropriado e intercultural, baseado na articulação e complementariedade entre saberes em saúde, vem sendo uma prioridade para garantir a atenção primária à saúde (APS) dos povos indígenas desde a Conferência de Alma-Ata. No Brasil, país de significativa variedade sociocultural no contexto indígena sul-americano, existe há 16 anos uma Política Nacional de Atenção à Saúde das Populações Indígenas (PNASPI) focada no conceito de atenção diferenciada. Esse conceito, considerado como incompleto e contraditório, é variavelmente operacionalizado na APS de indígenas. Sendo assim, o presente artigo propõe uma análise da formulação e operacionalização desse conceito na PNASPI. Essa análise torna evidente o caráter etnocêntrico da PNASPI, as numerosas contradições e negligências que não contemplam de fato o intercâmbio e articulação com o saber tradicional e as visões êmicas indígenas de saúde e dos processos de padecimento/cura. A reversão dessas limitações exigirá maior reflexividade, questionamento e vigilância epistemológicos tanto das ciências sociais e politicas quanto dos movimentos sociais e de controle social indígenas para redefinir em termos interculturais a APS de indígenas no Brasil.

Palavras-chave Saúde indígena; interculturalidade; política pública; atenção à saúde; Brasil.

As orientações da Declaração de Alma-Ata há 40 anos fundamentam o desenho dos modelos sanitários nacionais centrados na igualdade de acesso à atenção primária à saúde (APS). Considerada porta de entrada, coordenadora do cuidado e ordenadora das ações e serviços disponibilizados no Sistema

\footnotetext{
1 Universidade Federal da Bahia (UFBA), Instituto de Saúde Coletiva (ISC), Salvador (BA), Brasil. Correspondência: Leo Pedrana, leo.pedrana@ gmail.com

2 Fundação Oswaldo Cruz, Centro de Pesquisas Leônidas e Maria Deane, Manaus (AM), Brasil.
}

Único de Saúde (SUS), no Brasil a APS deve ser ofertada universal e gratuitamente, de acordo com as demandas e necessidades do território, e considerando os determinantes e condicionantes de saúde (1). Nos territórios indígenas do país, as ações de APS são ofertadas através do Subsistema de Atenção à Saúde Indígena (SASI), sob uma perspectiva participativa e intercultural, da qual os agentes comunitários indígenas e praticantes tradicionais participam como "importantes aliados" dos profissionais da atenção primária "na organização de medidas para aprimorar a saúde da comunidade" (2).

Como outras nações, o Brasil também busca garantir serviços de saúde pública universais, integrados, apropriados e compreensivos (3) e ações de cuidado culturalmente apropriadas, respeitando as especificidades e direitos socioculturais conforme a Convenção 169 da Organização Internacional do Trabalho (OIT) (4). Tais ações e serviços devem ser fundamentados na articulação com os saberes e práticas tradicionais indígenas em saúde através da introdução de novos 
conceitos nas políticas públicas, transformação das formas organizativas dos modelos de atenção e definição de novas práticas de cuidar. Não obstante, os indígenas seguem sendo considerados, em nível global, um dos grupos populacionais mais vulneráveis. $\mathrm{O}$ fosso entre as condições de saúde dos indígenas e aquelas das sociedades nacionais "envolventes" persiste, ou seja, a iniquidade na saúde indígena segue sendo um problema prioritário na agenda mundial (5).

Na América do Sul, a trajetória histórica de criação de diversos modelos e estratégias institucionais de APS para as populações indígenas, orientados pela interculturalidade, é marcada pela luta dos movimentos sociais indígenas e pela postura crítica pós-colonial das análises acadêmicas (6). Por sua vez, o Brasil, país de extensão continental e vasta heterogeneidade étnico-linguístico-cultural, incorporou o reconhecimento dos direitos diferenciados dos indígenas com a Constituição de 1988, mas ainda limitando esses direitos a aspectos sociais e culturais, deixando em segundo plano a garantia dos direitos à soberania, à autodeterminação e à representação no processo de tomada de decisões políticas, perpetuando resquícios de uma herança histórica de colonização e escravidão.

No campo do direito à saúde, o movimento indígena reivindicou, a partir da I Conferência Nacional de Proteção à Saúde Indígena, em 1986, um subsistema de atenção voltado às especificidades dos seus povos. Isso se efetivou anos mais tarde, através da "lei Arouca", com a criação de um modelo de atenção com enfoque integral e diferenciado (7), considerando que:

O processo de saúde/doença dos povos indígenas é o resultado de determinantes socioeconômicos e culturais, que vão desde a integridade territorial e da preservação do meio ambiente, à preservação dos sistemas médicos tradicionais desses povos e da preservação da cultura como um todo, da autodeterminação política e não somente pela assistência à saúde prestada. É da máxima importância observar a formação de recursos humanos adequados a prestarem assistência médico-sanitária aos povos indígenas, levando em conta o conhecimento e o respeito às medicinas tradicionais dessas populações, procurando estratégias de mudanças de postura etnocêntrica e estritamente tecnológica dos profissionais de saúde, em todos os níveis do país (Deputado Sérgio Arouca, Sala de Sessões, em 29 de junho de 1994) (8).

A "abordagem diferenciada e global" prevista na Política Nacional de Atenção à Saúde dos Povos Indígenas (PNASPI) (9, p. 28) estabelece a criação do SASI, definido como um subsistema "complementar e diferenciado" do SUS (9, p. 13), concebido com base em elementos de especificidade e diferenciação estruturados "sob a bandeira" da atenção diferenciada em contextos considerados interculturais (10).

Estudos empíricos que analisaram a operacionalização da PNASPI evidenciam um modelo de assistência que produz uma "normatização inclusiva" (11) e o predomínio de práticas assistencialistas e que hegemonizam as diferenças (12). Prevalece uma interculturalidade funcional, na qual "o reconhecimento e respeito à diversidade cultural se convertem em uma nova estratégia de dominação que ofusca e mantém, ao mesmo tempo, a diferença colonial através da retórica discursiva do multiculturalismo" $(13$, p. 4, tradução livre dos autores).

Assim, os estudos apontam que a operacionalização da PNASPI não tem favorecido a produção de ações em saúde afinadas com as lógicas culturais e as demandas efetivas das comunidades indígenas. Em realidade, tem produzido efeitos contrários aos princípios e pressupostos fundantes da política, especialmente no que diz respeito à adaptação e ao aperfeiçoamento do funcionamento e à adequação da capacidade do SUS às "necessidades específicas" de saúde das "comunidades culturalmente diferenciadas", referenciadas na "saúde diferenciada" (14).

A saúde coletiva e a antropologia coincidem ao enfatizar a opacidade e a complexidade do conceito de atenção diferenciada, refletidas tanto no texto da PNASPI quanto na sua implementação (15). Por outro lado, algumas análises se revelam pouco críticas frente às fragilidades apontadas, contribuindo para invisibilizar contradições, lacunas e pressupostos etnocêntricos presentes na referida política. Nesse contexto, o presente artigo se propõe a interrogar quais aspectos do complexo conceito de atenção à saúde diferenciada são claramente definidos na Política de Saúde Indígena
(9) em termos de operacionalização e quais são os pontos negligenciados ou contraditórios que (des)orientam o modus operandi dos profissionais da saúde.

Para abordar a questão da atenção diferenciada evoca-se a concepção de interculturalidade crítica de Catherine Walsh (16, p. 4-5), na qual encontramos categorias que podem ser referenciadas ao campo da saúde indígena: um processo de relação, comunicação e aprendizagem entre culturas a partir de condições de respeito, legitimidade mútua, simetria e igualdade; um intercâmbio entre pessoas, conhecimentos, saberes e práticas culturalmente diferentes para desenvolver um novo sentido entre elas na sua diferença; um espaço de negociação e tradução, onde as desigualdades e relações de poder da sociedade são reconhecidos e confrontados.

\section{"Diferenciado": sentidos e aplicações práticas na PNASPI}

O termo "diferenciado" se apresenta na PNASPI sobretudo em relação ao "modelo diferenciado de organização de serviços", considerando "formas ocidentais convencionais" (9, p. 1), com menor ênfase sobre as "práticas sanitárias" (9, p. 13). A preocupação com a "adequação" do sistema público de saúde aos sistemas tradicionais de saúde centra-se, portanto, sobre questões formais e "tecnologias apropriadas" (9, p. 6) em um sentido genérico, baseado na visão etnocêntrica de organização dos serviços.

Na diretriz relativa ao "monitoramento das ações de saúde", o habitus biomédico se reflete na preocupação em definir critérios e indicadores estritamente epidemiológicos como instrumentos que deverão "permitir a identificação dos riscos e das condições especiais que interferem no processo de adoecer" e "que avaliem a saúde e, indiretamente, a atenção à saúde" $^{\prime \prime}(9$, p. 16). De fato, o monitoramento da saúde indígena não é definido em termos interculturais: não prevê indicadores para monitorar as práticas interculturais de cuidado, nem tampouco considera a participação das comunidades indígenas na gestão, implementação e acesso aos dados (17). O Sistema de Informação da Saúde Indígena (SIASI) não é de público acesso e a sua implementação privilegia metas quantitativas sem considerar variáveis de natureza qualitativa da efetividade do cuidado intercultural.

Em outro trecho da PNASPI, aludese a "serviços diferenciados" (9, p. 15) na 
lógica da integralidade, aqui entendida como acesso e continuidade do tratamento entre níveis de complexidade diferentes, e "por intermédio de diferenciação de financiamento". O modelo de gestão do SASI se estrutura seguindo uma lógica piramidal na qual se inserem numerosas empresas privadas e organizações não governamentais, diferenciando o modelo de atenção desse subsistema no SUS em termos de maior dependência da terceirização na assistência: uma fragmentação que envolve desde os trabalhadores até os serviços essenciais como transporte sanitário e limpeza.

Os aspectos mais claramente orientados a uma perspectiva intercultural e indigenista da PNASPI, tais como a articulação entre saberes, respeito de práticas e valores do sistema tradicional e a consideração e reconhecimento da diversidade, constam na seção das diretrizes farmacológicas (9, p. 17). Essas diretrizes explicitam a valorização das práticas farmacológicas tradicionais como estratégia de articulação entre saberes fitoterápicos, através da "avaliação e adaptação dos protocolos padronizados de intervenção", além de ressaltar a questão do "controle de qualidade e vigilância em relação a possíveis efeitos iatrogênicos" $(9$, p. 18). No entanto, a dita articulação entre farmacologia ocidental e tradicional indígena fica aberta a leituras ambíguas: por um lado, manifesta-se uma preocupação pelo impacto da troca cultural de um objeto, o medicamento farmacológico e, por outro lado, aparece uma perspectiva unidirecional de apropriação de conhecimento, das práticas fitoterápicas e dos segredos do ofício da pajelança de tipo predatório, não complementar.

Fica claro que os elementos de diferenciação do SASI são definidos nas primeiras diretrizes da PNASPI com ênfase na formalidade da organização dos serviços. A substancialidade do cuidado diferenciado ofertado é atravessada por contradições, a partir da dominante visão biomédica da saúde e da atenção à saúde indígena.

A lógica monocultural e unidirecional subjacente à política nacional se revela também na diretriz "articulação dos sistemas tradicionais", na medida em que não prevê, como o próprio título sugere, o SUS como objeto de articulação com os demais sistemas médicos tradicionais, supostamente subalternos ou periféricos.

A natureza epidemiológica dos critérios que definem o perfil e as competências das equipes multiprofissionais de saúde indígena (EMSI), que operam na APS voltada a esse grupo, é outro aspecto que salta aos olhos, uma vez que sua composição e parametrização variam de acordo com "o número de habitantes, a dispersão populacional, as condições de acesso, o perfil epidemiológico, as necessidades específicas para o controle das principais endemias" $(9$, p. 14). Torna-se evidente a falta de referência às competências comunicativo-relacionais e linguístico-culturais necessárias para a mediação em contextos interculturais considerando as 274 línguas indígenas brasileiras (18).

Assim, o aspecto multidisciplinar da composição das EMSI se reduz a priori e $a$ fortiori quase que exclusivamente aos profissionais da saúde pública stricto sensu, reproduzindo exatamente o modelo de equipe da Estratégia Saúde da Família (ESF) do SUS não diferenciado. A definição organizativa do modelo de atenção na APS exclui, portanto, a contribuição de especialistas de outros saberes da saúde, como o cuidador tradicional indígena, ou campos e disciplinas acadêmicas orientadas à interculturalidade na saúde, pois a "colaboração sistemática de antropólogos" (9, p. 14) não se efetiva necessariamente na oferta cotidiana de atenção às comunidades indígenas do Brasil. A visão eurocêntrica da PNASPI considera os profissionais das EMSI como "brancos" e representantes da "medicina dos brancos", ocidental e biomédica. Isso ocorre apesar do aumento considerável, nos últimos anos, da profissionalização de indígenas que atuam na saúde indígena além dos agentes indígenas de saúde (AIS) (19).

Outra lacuna importante diz respeito à ausência de investimento em processos de formação diferenciados, estruturados com base no conhecimento tradicional do contexto local e competências relacionais e comunicativas interculturais, capazes de produzir relações de cuidado efetivamente diferenciadas (20). Embora a PNASPI reconheça a centralidade da formação "como instrumento fundamental de adequação das ações dos profissionais e serviços de saúde do SUS às especificidades da atenção à saúde dos povos indígenas" (9, p. 16), é prevista exclusivamente para uma categoria de profissional, o AIS, a partir de uma concepção que se arrisca em termos assimilacionistas e integracionistas como "uma estratégia que visa favorecer a apropriação, pelos povos indígenas, de conhecimentos e recursos técnicos da medicina ocidental".
Isso se reflete na hierarquização das relações nas EMSI, baseada no conhecimento especializado das patologias como clara demonstração do predomínio hegemônico da biomedicina na concepção de atenção diferenciada. Não está prevista a reciprocidade na apropriação e incorporação de outros saberes em saúde pelos outros profissionais das EMSI (médico, odontólogo, enfermeiros, técnicos e assistentes).

O percurso de compreensão do sentido de cuidado das comunidades assistidas e da aproximação ao processo de saúdedoença e ao saber tradicional em saúde não se dá com base em processos interculturais de troca, intercâmbio, reconhecimento recíproco, bi-direcionalidade e polissemia de práticas de fazer a saúde diferentes. Isso demonstra uma outra contradição com os pressupostos que fundamentam a lei nacional no que se refere a articulação e respeito entre saberes, uma vez que a sua operacionalização, paradoxalmente, não prevê a inclusão no sistema de saúde público dos especialistas tradicionais (pajés, rezadores, benzedores, raizeiros, parteiras).

\section{CONSIDERAÇÕES FINAIS}

A análise do texto da PNASPI evidencia uma visão intercultural funcional, mais que crítica e operacional, do fazer diferenciado em relação à atenção à saúde indígena, que passa pelos extremos do etnocentrismo até as contradições sobre os pressupostos da articulação entre saberes, central para a concepção de atenção diferenciada. Ao retomar como referência o conceito de inteculturalidade crítica, a análise evidencia os nós que viram obstáculos e barreiras para o entendimento de atenção diferenciada como um processo de interação mutuamente construído, compartilhado e validado. As ambiguidades ou ambivalências que permeiam a noção de atenção diferenciada se apresentam como empecilhos à construção de novas práticas interculturais de cuidar conforme as intenções expressas nas premissas fundantes da legislação nacional.

As conferências nacionais, expressões do ponto de vista de indígenas e sanitaristas indigenistas, seguem demandando e sugerindo estratégias interculturais como a inclusão dos especialistas tradicionais nos serviços de atenção à saúde indígena e uma formação intercultural para os profissionais não indígenas. Porém, em termos institucionais, a vocalização das visões êmicas indígenas sobre a saúde é 
fortemente limitada pelo caráter consultivo das instâncias de controle social indígena (conselhos e conferências nacionais de saúde indígenas) que por vezes constrange a concreta participação no processo de tomada de decisão sobre a saúde pública indígena.

A perspectiva crítica da política de atenção pode ser, nesse sentido, um meio estratégico para superar as barreiras erigidas pela tendência monolítica da biomedicina, expressão de hegemonia cultural sobre as silenciosas resistências e domínio dos adormecimentos dos milenares saberes comunitários indígenas.

Faz-se necessário empreender um exercício rigoroso de autorreflexão e de vigilância epistemológica capaz de construir um processo virtuoso de reflexão crítica intercultural que passa pelo reconhecimento institucional e científico das epistemologias do saber indígena em saúde e pela análise de práticas e saberes tradicionais comunitários de autoatenção (21), explicitando a visão êmica indígena sobre os processos de adoecimento e cura em uma perspectiva de múltiplos sistemas terapêuticos. Isso permite focar os processos de hibridismos e distinções entre as epistemes e vislumbrar possibilidades de articulação e integração entre as práxis de atenção à saúde indígena em um sentido intercultural. O processo de revisão da PNASPI que culmina com a realização da próxima $6^{\text {a }}$ Conferência Nacional de Saúde Indígena deve (re)considerar os fundamentos interculturais da noção de atenção diferenciada, tanto em nível de política como de episteme científica, e construir estratégias para efetivá-los nas práxis dos sujeitos que ofertam cuidado em saúde aos povos indígenas.

Ao considerar a reforma sanitária brasileira como um processo em construção (22), podemos pensar uma nova era de interculturalidade promovida pelo ativismo dos indígenas em sinergia com a pesquisa cientifica implicada, aplicada

\section{REFERÊNCIAS}

1. Brasil, Ministério da Saúde, Secretaria de Atenção à Saúde, Departamento de Atenção Básica. Política Nacional de Atenção Básica. Brasília: Ministério da Saúde; 2016. Disponível em: http://bvsms.saude.gov. $\mathrm{br} / \mathrm{bvs} /$ publicacoes/politica_nacional atencao_basica_2006.pdf Acessado em 12 de setembro de 2017.

2. World Health Organization (WHO). Primary health care: report of the International Conference on primary health care, Alma-Ata, USSR, 6-12 September 1978. Genebra: WHO;1978. Disponível em: http: / / apps.who.int/iris/bitstream / 10665/39228/1/9241800011.pdf Acessado em 10 de setembro de 2017.

3. Pan American Health Organization (PAHO). Renewing primary health care in the Americas. A position paper of the Pan American Health Organization/WHO. Washington, D.C.: PAHO; 2007. Disponível em: http://apps.who.int/medicinedocs/ documents/s19055en/s19055en.pdf; Acessado em 19 de outubro de 2017.

4. Brasil, Casa Civil, Subchefia para Assuntos Jurídicos. Decreto 5 051/2004. Disponível em: http://www.planalto. gov.br/ccivil_03/_ato2004-2006/2004/ decreto/d5051.htm Acessado em 19 de setembro de 2018.

5. Anderson I, Robson B, Connolly M, Al-Yaman F, Bjertness E, King A, et al. Indigenous and tribal peoples' health (The Lancet-Lowitja Institute Global Collaboration): a population study. Lancet. 2016;388(10040):131-57.

6. Silveira NH. Políticas públicas de saúde e indigenismo na América Latina. Em: Langdon EJ, Cardoso MD, org. Saúde indígena: políticas comparadas na América Latina. Florianópolis: Editora da UFSC; 2015.

7. Brasil. Lei $9836 / 1999$. Diário Oficial da União. 1999;24 set; Disponível em: http:/ / www.planalto.gov.br/ccivil_03/leis/L9836. $\mathrm{htm}$ Acessado em 10 de outubro de 2017.

8. Brasil. Projeto de lei 4681/1994. Disponível em: https://goo.gl/Rth5w7 Acessado em 3 de janeiro de 2013.

9. Brasil, Ministério da Saúde, Gabinete do Ministro. Portaria 254/2002. Diário Oficial da União. 2002;31 jan; Seção 1. Disponível em: http://bvsms.saude.gov.br/bvs/publicacoes/politica_saude_indigena.pdf Acessado em 10 de outubro de 2016.

10. Diehl EE, Langdon EJ. Transformações na atenção à saúde indígena: tensões e negociações em um contexto indígena brasileiro. Univ Humanist. 2015;80:215-36.

11. Cardoso MD. Saúde e povos indígenas no Brasil: notas sobre alguns temas equívocos na política atual. Cad Saude Publica. 2014; 30(4):860-6.

12. Pereira OP, Almeida TMC. Saúde e poder: um estudo sobre os discursos hegemônicos e subalternos em contextos multiculturais. Rev Bras Crescimento Desenvolv Hum. 2005;15(2):91-8.

13. Walsh C. Interculturalidad crítica y pedagogía de-colonial: apuestas (des) de el insurgir, re-existir y re-vivir. UMSA Rev (Entre Palabras). 2009;3.

14. Ferreira LO. A emergência da medicina tradicional indígena no campo das políticas públicas. Hist Cienc Saude-Manguinhos. 2013;20(1):203-19.

15. Pontes ALM, Rego S, Garnelo L. O modelo de atenção diferenciada nos Distritos Sanitários Especiais Indígenas: reflexões a e coletiva. Nesse sentido, esperamos que a análise proposta possa tornar-se um instrumento útil para concretizar novas estratégias de restruturação e indigenizacão da interculturalidade nas práticas de atenção à saúde no âmbito do subsistema público voltado às populações brasileiras originárias.

Agradecimentos. $\mathrm{O}$ estudo teve apoio do Conselho Nacional de Desenvolvimento Científico e Tecnológico (CNPQ) pela concessão de bolsa de doutorado ao primeiro autor.

Conflitos de interesse. Nada declarado pelos autores.

Declaração. As opiniões expressas no manuscrito são de responsabilidade exclusiva dos autores e não refletem necessariamente a opinião ou política da RPSP/PAJPH ou da Organização PanAmericana da Saúde (OPAS). partir do Alto Rio Negro/AM, Brasil. Cienc Saude Coletiva. 2015;20(10):3199-210.

16. Walsh C. La Interculturalidad en la educación. Lima: Ministerio de Educación, Dirección Nacional de Educación Bilingüe Intercultural; 2005.

17. Walker J, Lovett R, Kukutai T, Jones C, Henry D. Indigenous health data and the path to healing. Lancet. 2017; 390(10107): 2022-3.

18. Instituto Brasileiro de Geografia e Estatística (IBGE). Censo 2010. Disponível em https:// censo2010.ibge.gov.br/ Acessado em 11 de setembro de 2014.

19. Diehl EE, Pellegrini MA. Saúde e povos indígenas no Brasil: o desafio da formação e educação permanente de trabalhadores para atuação em contextos interculturais. Cad Saude Publica. 2014;30(4):867-74.

20. Langdon, EJ, Diehl EE. Participação e autonomia nos espaços interculturais de saúde indígena: reflexões a partir do sul do Brasil. Saude Soc. 2007;16(2):19-36.

21. Menéndez EL. Modelos de atención de los padecimientos: de exclusiones teóricas y articulaciones prácticas. Cienc Saude Coletiva. 2003;8(1):185-207.

22. Paim JS. Reforma sanitária brasileira: contribuição para a compreensão e crítica. Rio de Janeiro: Fiocruz; 2008.

Manuscrito recebido em 22 de dezembro de 2017 Aceito em versão revisada em 22 de agosto de 2018 . 
ABSTRACT

Critical analysis of interculturality in the National Policy for the Care of Indigenous Peoples in Brazil

Keywords
Concern for culturally appropriate and intercultural care, based on linkages and complementarity among health knowledges, has been a priority for ensuring primary health care for indigenous peoples since the Alma-Ata Conference. In Brazil, a country with significant sociocultural variety in the South American indigenous context, a National Policy for the Care of Indigenous Peoples (PNASPI) was established 16 years ago, focusing on the notion of differentiated care. This concept, considered incomplete and contradictory, has been variably operationalized in indigenous primary health care. Therefore, the present article proposes an analysis of the formulation and operationalization of this concept in PNASPI. The analysis brings to light the ethnocentric nature of PNASPI, the numerous contradictions and oversights that fail to encompass the exchanges and linkages with traditional knowledges and with indigenous emic views of health and processes of illness/cure. The reversal of these limitations will require greater epistemological reflexivity, questioning, and surveillance of both the social and political sciences, as well as social movements and indigenous social control, to redefine indigenous primary health care in Brazil in intercultural terms.

Health of indigenous peoples; cultural competency; public policy; health care (public health); Brazil.
RESUMEN

Análisis crítico de la interculturalidad en la Política Nacional de Atención a las Poblaciones Indígenas en Brasil

Palabras clave
La preocupación por un cuidado culturalmente apropiado e intercultural, basado en la articulación y complementariedad entre saberes en salud, es una prioridad para garantizar la atención primaria de salud de los pueblos indígenas desde la Conferencia de Alma-Ata. En Brasil, un país con una significativa variedad sociocultural en el contexto indígena de América del Sur, existe desde hace 16 años una Política Nacional de Atención a la Salud de las Poblaciones Indígenas (PNASPI) enfocada en el concepto de atención diferenciada. Este concepto, considerado incompleto y contradictorio, es ejecutado de manera variable en la atención primaria de salud de las poblaciones indígenas. Este artículo propone un análisis de la formulación y ejecución de ese concepto en la PNASPI. Este análisis hace evidente el carácter etnocéntrico de la PNASPI, las numerosas contradicciones y negligencias que no contemplan de hecho el intercambio y la articulación con el saber tradicional y las visiones émicas indígenas de salud y de los procesos de enfermedad/curación. La reversión de esas limitaciones requerirá mayor reflexividad, cuestionamiento y vigilancia epistemológica tanto desde las ciencias sociales y políticas como desde los movimientos sociales y de control social indígenas para redefinir en términos interculturales la atención primaria de salud de estas poblaciones en Brasil.

Salud de poblaciones indígenas; competencia cultural; política pública; atención a la salud; Brasil. 